\title{
Internet: entre las posibilidades de lo intangible, la dinámica social y las reconfiguraciones del capital
}

\author{
Horacio Edgardo Correa Lucero* \\ Julio Edgardo González**
}

Recibido: 22 de julio de 2014 - Aprobado: 16 de octubre de 2014

\section{Resumen}

En este artículo se esbozan elementos teóricos para el análisis de las nuevas tecnologías y en especial de Internet, dada la interrelación e influencia continua entre sociedad y tecnología. Ello permite abordar Internet como una serie de tecnologías digitales caracterizadas por un conjunto de capas imbricadas de carácter intangible y tangible. La hipótesis de este trabajo es que Internet ha puesto en crisis las formas clásicas de valoración del capital en relación a mercados signados directamente por precios y a la propiedad privada. De ahí que se concluya que los procesos en curso de configuración de la web sean producto de un entrecruzamiento entre las posibilidades que brindan los códigos técnicos en Internet desde sus inicios y la lógica del capital que, en cada caso particular, asume matices de ambas formas. De esto se sigue la importancia de analizar las particularidades del diseño y construcción de tecnologías, para comprender, a raíz de ello, las potencialidades y posibilidades políticas implicadas en los códigos técnicos del conjunto de tecnologías que componen Internet.

Palabras clave: tecnología, sociedad, Internet, capital, mercado.

* $\quad$ Máster en Estudios Sociales de la Ciencia y la Tecnología por la Universidad de Salamanca (España) y doctorando en Ciencias Sociales de la Universidad Nacional de Quilmes. Fue becario del programa de formación de la Fundación Carolina (España) y actualmente es becario de posgrado del Conicet (Argentina). Sus intereses de investigación incluyen los estudios sociales de la tecnología, redes sociales y economía política de la comunicación. Dirección postal: Roque Sáenz Peña 352, Bernal, Buenos Aires, Argentina. Correo electrónico: hecorrealucero@gmail.com

** Estudiante y becario de la Maestría en Ciencias Sociales y Humanidades de la Universidad Nacional de Quilmes (Argentina). Ex director de arte y diseño y editor en Wicked Magazine y Avantt Magazine. Ex director y creador de Avantt Media. Sus intereses de investigación incluyen los estudios sociales de la tecnología, la economía política de la comunicación, la teoría del actor red y el posestructuralismo. Dirección postal: Roque Sáenz Peña 352, Bernal, Buenos Aires, Argentina. Correo electrónico: juliogonzalezunq@gmail.com 


\title{
Internet: Among the possibilities of the intangible, social dynamics and reconfigurations of capital
}

\author{
${ }^{3}$ Horacio Edgardo Correa Lucero* \\ "Julio Edgardo González*
}

\section{Abstract}

In this article, theoretical elements for analysis of new technologies, especially the Internet are outlined, given the interrelationship and the continuing influence between society and technology. This allows to address the Internet as a series of digital technologies characterized by a set of tangible and intangible overlapping layers. The hypothesis of this study is that the Internet has placed the classical forms of capital in a critical situation regarding markets designated directly by private property and by prices. Hence, it is concluded that the ongoing processes of web configuration are the product of a cross-linking between the possibilities offered by technical codes on the Internet since its onset and the logic of capital that in each particular case assumes nuances of both forms. The result of this is the importance of analyzing the peculiarities of design and construction technologies, to understand, as a consequence, the potential and political possibilities involved in technical codes of the set of technologies that are part of the Internet.

Keywords: Technology, Society, Internet, capital market.

* Master in Social Studies of Science and Technology from the University of Salamanca (Spain) and PhD Doctorate in Social Sciences, National University of Quilmes. He was a fellow of the training program of the Carolina Foundation (Spain) and is currently a graduate fellow of CONICET (Argentina). His research interests include social studies of technology, social networks and political economy of communication. Address: Roque Sáenz Peña 352, Bernal, Buenos Aires, Argentina. Email: hecorrealucero@gmail.com.

** Student and fellow Masters in Social Sciences and Humanities, National University of Quilmes (Argentina). Former director of art and design and Wicked Magazine editor and Avantt Magazine. Former director and creator of Avantt Media. His research interests include social studies of technology, political economy of communication, actor network theory and post-structuralism. Address: Roque Sáenz Peña 352, Bernal, Buenos Aires, Argentina. Email: juliogonzalezunq@gmail. com. 


\title{
Internet: entre les possibilités de l'intangible, la dynamique sociale et les reconfigurations du capital
}

\author{
Horacio Edgardo Correa Lucero* \\ Julio Edgardo González*
}

\section{Résumé}

Cet article présente les éléments théoriques pour l'analyse des nouvelles technologies et notamment de l'Internet, compte tenu de l'interaction et de l'influence continue entre la société et la technologie. Cela permet d'aborder l'Internet en tant que série de technologies numériques caractérisées par l'ensemble de couches imbriquées de nature tangible et intangible.

L'hypothèse de ce travail consiste en ce que l'Internet a mis en crise les formes classiques d'évaluation du capital par rapport aux marchés signés directement par des prix et à la propriété privée. On peut conclure donc, que les processus web en cours de configuration soient le fruit d'un croisement entre les possibilités offertes par les codes techniques sur Internet depuis sa création et de la logique du capital qui, pour chaque cas individuel, suppose des nuances de ces deux formes. D'où s'ensuit l'importance d'analyser les particularités du dessin et de la construction de technologies, pour saisir, de ce fait, les potentialités et les possibilités politiques impliquées dans les codes techniques de l'ensemble de technologies composant Internet.

Mots clés: Technologie, société, Internet, capital, marché.

* Master en Études Sociales de la Science et la Technologie de l'Université de Salamanca (Espagne) et candidat au doctorat du programme Docteur en Sciences Sociales de l'Université Nationale de Quilmes. Bouisier du programme de formation de la Fondation Carolina (Espagne) et actuellement boursier du master du CONICET (Argentine). Ses intérêts de recherche comprennent les études sociales de la technologie, les réseaux sociaux et l'économie politique de la communication. Adresse: Roque Sáenz Peña 352, Bernal, Buenos Aires, Argentina. Email: hecorrealucero@gmail. com.

** Étudiant et boursier du Master en Sciencies Sociales et Humaines de l' Université Nationale de Quilmes (Argentine). Ex directeur d'art et dessin, éditeur chez Wicked Magazine et Avantt Magazine. Ex-directeur et créateur de Avantt Media. Ses intérêts de recherche comprennent les études sociales de la technologie, de l'économie politique de la communication, la théorie de l'acteur-réseau et le poststructuralisme. Adresse: Roque Sáenz Peña 352, Bernal, Buenos Aires, Argentina. Email: juliogonzalezunq@gmail.com. 


\section{Fundamentación teórica y metodológica}

Internet se manifiesta como un conjunto múltiple de tecnologías que, en su diseño, encierra una serie de limitaciones, como así también, de potencialidades. Al no presentar un control centralizado, Internet habilita la utilización de diversas plataformas y sitios web como vías de promover o contribuir a la participación política. Sin embargo, este conjunto de tecnologías y elementos abarcados por Internet habilita el pensamiento político y crítico sobre su propia construcción y, en definitiva, sobre las propias posibilidades políticas de la red de redes. En otras palabras, al comprender que la tecnología es política, observamos que ella misma (su propio diseño y construcción) implica actos políticos. De esto se sigue, a nuestro entender, la importancia de analizar las particularidades del diseño y construcción de tecnologías, para comprender, a raíz de ello, las potencialidades y posibilidades políticas implicadas en los códigos técnicos del conjunto de tecnologías que componen Internet. Dada la creciente expansión de Internet en países latinoamericanos (y las políticas emprendidas para su mayor expansión territorial en estas latitudes), consideramos clave poder contribuir en esta elucidación teórica.

Las producción teórica sobre dinámica (o cambio) social e Internet es amplia y presenta un crecimiento sustancial en los últimos años, tanto en la literatura anglosajona, como en aquella publicada en nuestro idioma. Desde todos los sectores del amplio espectro de las ciencias sociales se han realizado estudios; algunos observan las posibilidades para el desarrollo social (Finquelievich y Prince, 2007), la inclusión social (Amariles, Paz, Russell, y Johnson, 2006; Parkinson y Lauzon, 2008; Warschauer, 2003), o el desarrollo comunitario (Gómez y Barón Porras, 2010; Unwin, 2009), donde Internet es usualmente observada (directa o indirectamente, consciente o inconscientemente) como una herramienta para el desarrollo y el bienestar social, como si las políticas de acceso a la capa tangible de Internet (hardware, incluyendo infraestructura) fuera condición necesaria y suficiente para garantizar mejoras sociales. Otros analizan las potencialidades para el cambio social que ofrece Internet, comprendiendo las modificaciones al repertorio político que esto significa para los movimientos sociales (Earl y Kimport, 2011), o bien, los modos en que los seres humanos lo usan para cambiar las instituciones sociales y, en caso de concebir que esos cambios suceden, para analizar si los mismos son graduales o si implican transformaciones de los modos previos de organización (Surrat, 2001). En una línea similar, Micah White propuso el término "clicktivismo" para sostener que el activismo basado en firmar peticiones online, o en dar "clics" a diversas campañas, simplemente arruina el activismo de izquierda, y toda posibilidad de transformación radical de la sociedad. El uso de estrategias de mercadeo y de publicidad en este tipo de campañas políticas, alimenta esas lógicas, en detrimento de la política (White, 2010).

En estas visiones, suele dejarse de lado un aspecto de vital importancia para la compresión política de las tecnologías digitales y de Internet en particular: su diseño y construcción y, consecuentemente, sus códigos técnicos. Si bien todas 
ellas tienen elementos de importancia para el análisis político de Internet, de ellas, si bien no es algo que utilicemos en el desarrollo teórico, la propuesta de Micah White puede encerrar algo de mayor utilidad. A nuestro entender, permite visibilizar el modo en que el capitalismo busca subsumir todo bajo su lógica, incluso el activismo político; uno de los modos en que describiremos esto será con la afirmación de que el capitalismo busca la creciente "mercantilización de todas las cosas" (Wallerstein, 1988, p. 4).

En concreto, dicho esto, podemos afirmar dos cuestiones centrales. Por un lado, nuestra intención de señalar el modo en que el diseño y la construcción de tecnologías digitales encierra una importancia central en términos políticos. Por el otro, nuestra visión acerca de la necesidad de considerar una estructura social más amplia, como el capitalismo, para visibilizar las potencialidad políticas de las tecnologías (y sus sesgos). Comprender dónde estas se inscriben y las tensiones por su apropiación, devienen temas centrales para el análisis teórico. El primer elemento lo desarrollamos en el punto 2, y partir de él, surgirá la necesidad de comprender los principios teórico-técnicos de Internet como conjunto de tecnologías digitales, algo que desarrollaremos en el punto 3. Luego de ello, en el punto 4, estaremos en condiciones de exponer las tensiones emergentes en Internet y sus nexos con las potencialidades para el cambio social.

Antes de comenzar, conviene realizar algunas aclaraciones. El presente artículo es parte de una investigación mayor centrada en el caso de YouTube en su relación con la Industria Cultural, desde sus inicios en el año 2005 hasta el presente. Por razones operativas la hemos divido en dos partes fundamentales. La primera, presentada aquí, consiste en el relevamiento teórico para desplegar el análisis empírico. Hemos realizado una propuesta teórica para analizar las tecnologías digitales e Internet en particular. Por otro lado, la segunda parte de la investigación (trabajo que hemos comenzado a realizar y concluiremos en los próximos meses, razón que explica su no inclusión aquí), implica el relevamiento de información sobre nuestro caso de estudio, YouTube. Las fuentes, en este caso, son secundarias (noticias y libros publicados sobre YouTube). En una segunda etapa, incluiremos entrevistas orales a ejecutivos de la firma en Argentina (incluyendo ejecutivos de Google Inc.).

De este modo, la parte aquí publicada, puede ser caracterizada como investigación exploratoria, basada en fuentes secundarias y de corte netamente teórico. En este sentido, presentamos en el presente artículo una propuesta de abordaje teórico de Internet, como paso fundamental para poder analizar las potencialidades políticas de la red de redes. Así, un intento de aprehensión dialéctica de las tecnologías digitales es lo que guía, en un comienzo, nuestro trabajo teórico. De esta manera, comenzamos brindando una visión abstracta de las tecnologías digitales, hasta llegar a un nivel de concreción donde estas aparecen con todas sus determinaciones socio-técnicas. Así llegaremos a analizar la fragmentación por capas que caracteriza a Internet y, a partir de allí, desprendemos una serie de actores que participan de uno u otro modo en esos "espacios" de Internet. 
Nuestras unidades de análisis son: Internet (como espacio conformado por un conjunto de tecnologías digitales), sujetos (que surgen en cada capa de Internet: empresas, activistas, desarrolladores de software, usuarios/productores). Nuestro objeto de estudio se encuentra en la comprensión teórica de Internet y de sus potencialidades políticas, a raíz de su inserción en un contexto social más amplio (el capitalismo).

\section{Tecnología y sociedad: conceptos de relevancia ${ }^{1}$}

Andrew Feenberg entiende, siguiendo a autores constructivistas, que el diseño y construcción de objetos técnicos es emprendido por grupos sociales interesados en tales procesos. Esos grupos realizan prácticas a través de lo que la tecnología significa para ellos, pero, a su vez, ese significado influye en sus desarrollos tecnológicos. Tales grupos forman parte del horizonte cultural de la sociedad en la que están circunscriptos (Feenberg, 2010, p. 16). El horizonte cultural refiere a supuestos culturales generales e incuestionables que están en la vida social misma y que son manifestación cultural de relaciones de poder dentro de una sociedad. Expresado de otro modo, son meras formas hegemónicas naturalizadas (Feenberg, 2010, p. 16; Gramsci, 1986). El horizonte cultural de la tecnología moderna está asentado en la racionalidad (en el creciente cálculo y en el control de la vida social) (Weber, 1998) y en la eficiencia técnica; pero a su vez se encuentra limitado por normas de la cultura y la tradición. Estos valores cristalizan en la estructura de las máquinas y en su diseño (Feenberg, 2010, p. 17) estableciendo códigos técnicos, los cuales atraviesan una serie de disputas tecnológicas, culturales y políticas hasta que se concretizan. Esto indica que para cualquier problema hay distintas soluciones posibles en función de las interpretaciones de los diversos grupos que buscan definir las tecnologías de acuerdo a sus propios significados (Bijker, 1997). Esta perspectiva ha entendido que existen tantos artefactos como grupos sociales relevantes hayan en el momento en que una tecnología se encuentra en proceso de construcción. De este modo, las innovaciones deben conciliar diversos factores contextuales. El código técnico deja entrever que la eficiencia no es el único valor en juego en la construcción de tecnologías (Feenberg, 2010, p. 66). Eso llamado racionalidad en la tecnología tiene algo de fiasco inmanente, debido a que se presenta como el único y fundamental elemento en el diseño de las tecnologías, sin serlo.

Consagrada la visión de un grupo, es decir, superadas las controversias, la tecnología se cristaliza y se consolida durante un tiempo prolongado (Feenberg, 2010, p. 10) y, a la vez, actúa validando e imponiendo un orden, un sesgo (eliminando

1 El desarrollo teórico aquí expuesto es una concepción aún en desarrollo. Tal como hemos expuesto en la fundamentación teórica y metodológica, esta visión teórica es resultado de la primera parte de nuestro trabajo de investigación. A partir de lo desarrollado en la segunda parte (empírica y aquí no publicada por encontrarse en realización) estos conceptos serán profundizados. Por todo esto, la exposición aquí realizada representa un avance de nuestra investigación teórica. 
contingencias) a la sociedad, en tanto este horizonte cultural dominante se presente inconsciente a ella (Feenberg, 2010, p. 18).

El objeto técnico, en su construcción, debe cumplir ciertas funciones para resolver problemas específicos, esto acota certeramente las normas de su uso técnico, sin embargo, "los ingenieros no tienen control de todas las variables sociales que atraviesan a las tecnologías, ni de sus efectos sobre las sociedades y los patrones de vida" (Feenberg, 2010, p. 23). El uso de tecnologías instaura conflictos, controversias y nuevas demandas. Originan resistencias que pueden traducirse en nuevos códigos técnicos. Feenberg afirma que entre tecnología y sociedad existe una comunicación constante mediada por la realización de valores en el diseño y el impacto del diseño en los valores. Aquello que Latour denomina "delegación" (Latour, 1992). De este modo las tecnologías encuentran sustento para poder mantener su hegemonía en el tiempo (Feenberg, 2010, p. 68).

Los objetos técnicos son atravesados por la sistematización y mediaciones valorativas. Con sistematización Feenberg se refiere a:

El sistema de significados socialmente establecidos que determina la naturaleza de las tecnologías y las interconexiones entre sus diversas partes y sus entornos técnicos, humanos y naturales. Con mediaciones valorativas se refiere a los aspectos que gobiernan las tecnologías que incluyen criterios éticos, estéticos y otros normas sociales generales (Feenberg, 2010, p. 73).

La interacción de estos dos aspectos (...), es un proceso iterativo en el que el significado que las tecnologías asumen en la vida se alimenta en su diseño de una etapa de su desarrollo a la siguiente (Feenberg, 2010, p. 73).

Siguiendo ideas de Miki (1967) la tecnología es "subjetivo-objetiva." Por un lado requiere de la subjetividad para su creación y, por otro, se objetiva en una forma concreta externa que se nos enfrenta como una realidad tangible. De este modo, Miki explica a la historia como un "movimiento" que no puede escindirse de la creación tecnológica (Miki, 1967, p. 211).

En definitiva, Feenberg sostiene que, en su trayectoria, las tecnologías satisfacen necesidades creando nuevas necesidades y con esto, co-construyen nuevos seres humanos y sociedades (Feenberg, 2010, p. 146). Es decir, ninguno de los elementos es determinante unidireccionalmente del otro.

Finalmente, Feenberg añade la necesidad de considerar el contexto social más amplio donde se despliegan las tecnologías. Este es el sistema capitalista, la lógica de valorización del capital, como elemento central para comprender los procesos de desarrollo y diseño tecnológico. Aquí, el conjunto de valores presentes en el ambiente de desarrollo de ciertas tecnologías específicas, reproducen o intentan evadir, la lógica mercantil. 


\section{Lo digital intangible y tangible}

Con el fin de llegar a una aprehensión teórica de lo que es Internet y de alguno de sus elementos componentes, creemos que debe realizarse un repaso de lo que consideramos esencial en la red de redes: las tecnologías digitales. Si bien es un texto de ciencias sociales, la explicación técnica es necesaria, a nuestro entender, debido a que de ello se siguen implicaciones teóricas de vital importancia para la comprensión de Internet.

Lo digital es, en primera instancia, en términos abstractos, un ente conformado por bits, esto es, dígitos binarios. Esos bits representan una esencia, una permanencia (en tanto haya alguna forma de vida o máquina que pueda interpretarlas y alojarlas) puramente abstracta propia de este tipo de bienes. El ordenamiento de bits es, de algún modo, eterno. Su naturaleza es la del número, y como tal, lo digital se encuentra más allá de lo real, de lo aprehensible, ya que, en tanto número, es ontológicamente ideal.

Este ente ideal, abstracto, que representan los bits, se inscribe en una totalidad donde cobra sentido técnico. Así, la sucesión ordenada de 1 y 0 es lo que la máquina o dispositivo físico (hardware) puede leer directamente. El sentido de "lectura" por parte de la máquina, implica la existencia de un lenguaje, de ahí el nombre dado a estos bits ordenados: lenguaje máquina o código objeto. Estos lenguajes son empleados para poder darle órdenes a las máquinas, sacarles provecho o incrementar, incluso, sus funcionalidades técnicas. Los existe de diferentes niveles: lenguaje máquina o lenguaje binario (lidian directamente con 1 y 0 ), lenguajes de programación o lenguajes de alto nivel (constituyen el código fuente). Finalmente, entre ambos, se encuentran los traductores, usualmente conocidos como ensambladores, compiladores o intérpretes (necesarios debido a que los dos primeros lenguajes no hablan el mismo "idioma").

Más allá de estos lenguajes, las instrucciones siguen una lógica algorítmica. Es decir, los binarios representan instrucciones realizadas mediante el desarrollo y utilización de algoritmos. El lenguaje binario (en conjunto con el hardware) brinda también la posibilidad de almacenar y reproducir datos o información de cualquier tipo y, además, de representar formas visuales, auditivas o sonoras.

En este proceso, la parte física o tangible está dada, en el caso, por ejemplo, de un disco rígido, por las posibilidades de dotar de carga positiva o negativa a los cabezales magnéticos del disco donde se almacenan estos datos, utilizando la codificación propia del lenguaje binario. A nivel físico podemos encontrar, ya en los chips y microchips, también un flujo de electrones movilizado con dos niveles de voltaje, representando cada nivel, nuevamente los 1 y 0 propios del lenguaje binario. Esto nos permite visualizar que la estructura binaria está presente tanto en la arquitectura del hardware, como en la del software (o componente intangible del bien digital).

En consecuencia, abstrayendo lo intangible, es posible hallar la materialidad técnica donde todo lo anterior (los números, los algoritmos, las cargas 
eléctricas) funciona, es decir, donde las cargas eléctricas se movilizan. En tanto materialidad, lo digital ya no aparece como eterno o inaproximable, sino como su opuesto. La materialidad implica un desgaste permanente y un camino sin retorno hacia la obsolescencia.

En la tecnología digital, lo intangible y lo tangible, pueden convivir en un dispositivo o, estar tan distanciados como geográficamente sea posible. En el primer caso puede tratarse de una computadora aislada, donde todos estos procesos funcionan en el nivel de la CPU, sin olvidar la comunicación con sus periféricos; en el segundo, de cualquier dispositivo conectado a alguna red o a la WWW, donde los procesos ocurren tanto a nivel local como en la red.

Ahora bien, una vez presentado lo digital en términos intangibles, es tiempo de incorporar los elementos sociales intervinientes en el análisis. Así, puede sostenerse que, en su unión, estos dispositivos físicos y numéricos (o intangibles) encuentran una funcionalidad técnica en la sociedad. El sentido que estos dispositivos digitales poseen, se vincula, en consecuencia, directamente con la sociedad donde funcionan y ella misma le implica una serie de restricciones a sus posibilidades técnicas ${ }^{2}$. Esto es, existe una estructura social que marca los límites del desarrollo contingente de los dispositivos técnicos.

Ambos elementos, lo tangible y lo intangible, son centrales para la comprensión de los modos en que se estructura Internet. La propia posibilidad de escindir ambos elementos es la base de la construcción de la red de redes. Estos mismos elementos, permiten una estructuración primaria de Internet en dos grandes capas, la intangible y la tangible. En la primera encontramos el software, los protocolos, la web, las redes sociales, etc. En la segunda, encontramos los espacios técnicos donde esos intangibles funcionan.

La estratificación, a partir de este momento, puede ser ampliada, vista en mayor o menor detalle, en cualquier caso, siempre preferimos utilizar definiciones que no consideren al objeto digital como monocapa (Latour y Zadunaisky, 2008, p. 209). En cuanto a otras visiones podemos citar tanto a Ariel Vercelli (2009), quien sostiene que existen tres capas: material, lógica y de contenidos (Vercelli, 2009, pp. 75-76), como a Mariano Zukerfeld quien propone cinco capas fundamentales: infraestructura, hardware, software, de contenidos $y$, finalmente, red social (Zukerfeld, 2010).

Las diferencias son menores entre estos elementos, solo establecen matices. Lo importante es que los dos grandes aspectos de las tecnologías digitales se manifiestan en esa misma estratificación: por un lado hallamos una parte intangible y, por el otro, una tangible o física. Según nuestra caracterización, en la capa intangible (lógica y de contenidos) funcionan las tecnologías y contenidos digitales intangibles de Internet, esto es, redes sociales, software, protocolos, aplicaciones,

2 Para un desarrollo en detalle acerca de las limitaciones en la conformación de las propiedad técnicas, en el diseño y construcción de una tecnologías que ofrece el sistema social donde funciona (el capitalismo) ver Söderberg, 2010. 
programas, contenidos digitales: música, fotos, videos, textos, etc.). A estos elementos los llamaremos, en forma intercambiable, bienes digitales de Internet.

Dentro de las ciencias sociales, la emergente disciplina de los Estudios de Internet (o Internet Studies) presenta análisis relativos a las capas de contenido y de software fundamentalmente (sin nombrarlas de ese modo), dejando a un lado el otro componente central de las tecnologías digitales, lo tangible o el hardware. Para nosotros, todo contenido que existe en Internet, funciona en todas esas capas. Si una falla, el bien de Internet no funciona, por lo tanto, de uno u otro modo, esos elementos deben considerarse.

\section{Tensiones en Internet}

Los elementos que constituyen (en sus vínculos) la capa digital intangible de Internet (información digital, aplicaciones, programas y software), en su producción y circulación social, pueden originarse o manifestarse en relaciones que tiendan a no reproducir las formas capitalistas ancladas en la relación capitaltrabajo, el mercado y la propiedad privada. Lo anterior no es una probabilidad vacía (o quizá no lo sea solo en cierto sentido) ${ }^{3}$. La diversidad de dispositivos que consolidan su producción (computadoras, teléfonos con wifi, tablets, etc.) se halla (en el momento de nuestra escritura) inmersa en las geografías, los sentidos y los cuerpos humanos, derramando así lo digital intangible desde/hacia variados planos drásticamente. Su creciente penetración afecta individualidades e intersubjetividades, originando, reconfigurando y (re)creando conexiones sociales (Wittel, 2012, p. 317). Estas máquinas digitales, soportes de materialidad sólida, funcionan, junto a lo digital intangible, como un nudo borromeo que puede disolver las fronteras entre agenciamientos fundamentales de la humanidad: el producir y el consumir. De este modo, la posibilidad técnica de subvertir la lógica del capital se introyecta en una creación cultural de potencial universal y de manifestación instantánea (Lessig, 2004, pp. 21-54-251).

Sin embargo, tal como sucede con todo objeto que, en su trayectoria histórica, haya sido resignificado (o producido) como mercancía para el capital, las creaciones digitales intangibles se enfrentan (además de a las necesidades de acumulación del capital, cuyo único límite es aquello no valorizable) a un dominio legal y tradicional que es el territorio en el cual se inscribe el poder ideológico intrínseco a las sociedades capitalistas (Aouragh, 2012, p. 524; McGuigan, 2012, p. 427).

Los hombres hacen su propia historia, pero no la hacen a su gusto, no la hacen bajo circunstancias elegidas por ellos, sino bajo circunstancias directamente encontradas, dadas y transmitidas desde el pasado (Marx, 1972, p. 10).

3 Si se tiene en cuenta la variedad de capas tangibles en Internet, en toda producción de bienes en la red, intervienen en varios modos la relación capital-trabajo. 
Este dominio se cimenta en una específica concepción de la propiedad de las cosas: la propiedad privada. Tal forma de propiedad no solo se relaciona a objetos, sino también a los sujetos, en cuanto su carácter privativo se inscribe respecto al no uso o copia de los mismos por un otro no propietario (Wittel, 2012, p. 329). La naturalización de la propiedad privada es una hendidura fundamental en el contenido subjetivo de los mundos mentales inmersos, de las representaciones sociales en las regiones de dominio capitalista.

Diversos elementos han incidido en una Internet no subsumida a la lógica de valorización del capital desde sus inicios ${ }^{4}$. Entre ellos, podríamos citar su origen académico-militar, los indispensables recorridos temporales de difusión de cualquier tecnología en una sociedad o, simplemente, múltiples y añejas desilusiones empresariales.

Sin embargo, la exigua importancia que las empresas capitalistas pudieron haber conferido a Internet en sus comienzos se encuentra en radical desvanecimiento, se evapora a través de su historia. Internet, en todas sus capas, presenta crecientes posibilidades de valorización del capital. En la gran red operan plenamente formas capitalistas transformando a la misma en una tecnología operativa a sus intereses hegemónicos (Schröter, 2012, p. 303). Esta tendencia del capitalismo de mercantilizar todo, se inscribe, si bien con algunas variaciones, en el paisaje narrativo de diversos autores (Adorno y Horkheimer, 2007; Hall y Stahl, 2012; Jameson, 2011; Lefebvre, 1974; Marx, 1973; Mosco, 2010; Peekhaus, 2012; Prodnik, 2012).

"El desarrollo histórico del capitalismo ha implicado una tendencia a la mercantilización de todas las cosas" (Wallerstein, 1988, p. 4).

Cabe aclarar (otra vez), que mercantilizar nuevas cosas es también resignificarlas, transformarlas en sí y en su relación con el/los mundo/s humano/s. El capitalismo, al expandirse, al convertirse en algo cercano a una totalidad que fluye influyendo, ejerce un movimiento contradictorio. Mientras más es, más pulveriza eso que es, eso que ocupa. Mediante la propiedad privada fragmenta los espacios, es decir, cada fragmento priva su propiedad (Hardt y Negri, 2002, 2006; Lefebvre, 1974, pp. 221-224; Prey, 2012).

Resituándonos en la materialidad sólida, el soporte es la interfaz entre el/los objeto/s digital/es intangible/s y su utilización con el fin de satisfacer necesidades y deseos humanos. Y además es, de acuerdo a su código técnico, aquello que ubica a las formas privativas y excluyentes del capitalismo clásico en una inexorable crisis, dado que la capa intangible de Internet no presenta limitaciones tecnológicas o económicas para su reproducción emancipatoria. De nuevo, pretender aplicar la propiedad privada (copyright tradicional) sobre la capa intangible de Internet es simplemente desconocer el sesgo que introdujo su código técnico original.

4 Recordemos que Internet se origina a partir de Arpanet, una red diseñada por la agencia militar estadounidense DARPA. En ella trabajaron científicos y tecnólogos académico-militares. 
Ante cualquier intención de signar la mediación de objetos intangibles creados en Internet a través de precios de mercado, tal agenciamiento suscitará diversas (re)acciones (Lessig, 2004, p. 217). En primer lugar, en el marco de la republicana aceptación, respeto (o devoción) por la legalidad, emergerán, tanto creaciones alternativas de entorno abierto como competencias intercapitalistas asentadas en modelos de negocios de bienes libres y gratuitos, por dar solo dos ejemplos. A la vez, ante la prohibición amenazante de herramientas como el copyright o el derecho de autor, diferentes usuarios harán valer su legítimo e ilegal "derecho a la resistencia", el ius resistentiae, con el fin de salvaguardar sus formas de vida y costumbres afirmadas (Virno, Estop, y Santucho, 2003). Diversas transgresiones, tales como la piratería, el cracking, el hacking (Lessig, 2004, p. 176) son prácticas culturales difundidas (no exentas de contrasentidos y de particularidades) (ver Lessig, 2004; Stallman, 2004; Vercelli, 2009). El copyright y el derecho de autor, tal como se manifiestan hoy (el marco legal ha cambiado su espíritu desde sus orígenes (Lessig, 2004, pp. 20-30-105-106-159-161)) de la mano de los requerimientos de la Industria Cultural, interfieren en "las formas habituales en las cuales los individuos crean y comparten la cultura" (Lessig, 2004, p. 21). Es la presencia de un marco regulatorio que viola las necesidades sociales y a sus poseedores. La propiedad privada en Internet gira en torno al copyright, ya que cada obra que se comparte automáticamente crea una copia (Lessig, 2004, p. 164). El copyright le da a su dueño el derecho a decidir los términos bajo los cuales se comparten (o valorizan) sus contenidos ${ }^{5}$ (Lessig, 2004, p. 82).

Sería imposible la ciencia si ella funcionara como funciona la Industria Cultural: "Perdone, Profesor Einstein, pero, ¿me podría dar permiso para usar su teoría de la relatividad para demostrar que usted estaba equivocado por lo que respecta a la física cuántica?" (Lessig, 2004, p. 41).

Sin embargo, lo precedente no imposibilitó la proliferación de empresas capitalistas en Internet. Por el contrario y, como ejemplo, diversos estudios revelan una alta concentración (en términos de visitas, páginas vistas, tiempos de uso, etc.) en un ínfimo número de firmas (en su mayoría empresas capitalistas) respecto al dominio de la World Wide Web. Lo que intentamos presentar, en realidad, son las limitaciones que el código técnico original (y los posteriores) de Internet impone a las clásicas formas de valorización directa del capital por medio de precios entre productores y consumidores (Lessig, 2004, p. 216). El escenario existente (y verificable), favorece, principalmente, a una propensión hacia un modelo de negocios de plataformas sociales (Google, Facebook, Twitter, YouTube) de conexión y distribución de subjetividades y objetos intangibles culturales altamente concentrado. El valor de uso de tales plataformas se asienta en la potencialidad de inclusión/exclusión de las conexiones y distribuciones subjetivo-objetivas que las mismas posibilitan y controlan (Prey, 2012, pp. 253-255). Es decir, permiten a sus usuarios producir "redes de significados, universos simbólicos y campos semánticos" con el fin de asociarse (Fisher, 2012, p. 177). En esta fluidez virtual

5 Es cierto que hay distintos tipos de copyright, algunos, minoritarios, permiten grados de uso más razonables, por ejemplo: Creative Commons (Lessig, 2004, p. 312) o Copyleft. 
en la cual acontece crecientemente el tiempo de la vida social, también se instaura el valor de cambio de las plataformas, relacionado a la capacidad de controlar y obtener datos susceptibles de valorización de las asociaciones/conexiones que potencian. Es decir, de su poder de adquirir información semántica y demográfica detallada tipeada por las propias fuentes subjetivas que las conforman. Esta información es un insumo fundamental del ciclo de valorización del capital y permite identificar, crear o reconfigurar nuevas prácticas consumistas de bienes de mercado (Fisher, 2012, p. 177). Esta información, desde otra mirada, lleva incrustada las normas y reglamentaciones impuestas por los sistemas que permiten su existencia (Prey, 2012, p. 255). Tal como afirmaron Jhally y Sut: "el valor de cambio de las mercancías culturales domina a su valor de uso" (Jhally y Sut, 1989). La mercancía de estas empresas es el consumo potencial de sus audiencias o usuarios, tal como planteó Dallas Smythe (1977) en su teoría de la triada de "medio-audiencia-anunciante" (Ekman, 2012, p. 166; Fisher, 2012, p. 172; Mosco, 2010, p. 137; Nixon, 2012, p. 450; Prodnik, 2012, p. 290; Smythe, 1977).

Desde nuestra perspectiva, el análisis teórico de Smythe es útil para comprender el trasfondo de mercantilización en la Industria Cultural. Sin embargo, consideramos que en el caso de plataformas sociales, esta triada no tiene tres partes. Es más, sería extraño hablar de un número específico de actantes. Si bien es un tema para profundizar en otro trabajo, entendemos que en el proceso de producción y mercantilización que, en la actualidad es controlado en gran medida por escasas plataformas sociales (ya mencionadas), participan infinidad de agentes desde diversos roles, influencias o jerarquías. Entre ellos podemos citar: las audiencias, los productores de contenidos culturales, los anunciantes, las empresas publicitarias, las plataformas y los dispositivos digitales tangibles.

A este punto de la trayectoria narrativa, deberían resultar perceptibles dos formas de creación o producción de objetos intangibles en Internet circunscriptos bajo valores diferentes, aunque aproximables. La primera de ellas, es la establecida por el código técnico inicial y los primeros usos de Internet, relacionados con la creación colaborativa, acumulativa, libre y gratuita. La otra forma es la de la producción de valores de mercado asociados a la propiedad privada (copyright y derechos de autor tradicionales), signados por precios. Ciertamente, estas formas, las cuales pueden actuar como límites, solo sirven para establecer las fronteras de una cartografía de las múltiples realidades de creación o producción en Internet, las cuales, no pueden ser más que específicas y diversas combinaciones de algunas de las características de estos límites infranqueables de la Internet actual.

Es por esto que cualquier producción social que tenga alguna característica marcada, sea un producto industrial, un verso, una fórmula, una idea política que apareció un día en alguna parte en un rincón de un cerebro, sueña como Alejandro con conquistar el mundo, trata de multiplicarse por miles y millones de copias en todas partes donde hay seres humanos y nunca se detendrá salvo si es contenida por una producción rival tan ambiciosa como ella (Tarde, 1895; 1999, p. 96 en Latour y Zadunaisky, 2008, p. 32). 
Es innegable que la capa intangible de Internet se ha derramado sobre cada ámbito de lo social (que disponga de soportes y conexión), incluyendo, por ejemplo, tanto a la producción y comercialización de bienes materiales (Hardt y Negri, 2006), como a los Estados, debido a las drásticas reducciones económicas que implican, entre otras de sus virtudes.

No obstante, la autonomía de la capa intangible de Internet respecto a la relación capital-trabajo y a la valorización del capital, se enfrenta a una serie de inconvenientes por su distanciamiento de los mercados. El compromiso con un proyecto de producción de bienes digitales intangibles (información digital, aplicaciones, programas y software) de distribución libre y gratuita implica, si nos place contemplar la existencia de temporalidad y la necesidad de subsistencia humana, la obligatoriedad de que los productores puedan financiarse por otros medios. Las desventuras económicas son un importante incentivo que influye en que cualquier producción colaborativa en Internet desemboque en un isomorfismo radical que conduzca hacia formas capitalistas o perezca.

Además, dado el ineludible requerimiento de capas tangibles para desarrollar cualquier objeto intangible de Internet, cualquier emprendimiento que desee extender su existencia debe cumplir con ciertos requisitos que demanda el actual desarrollo de la World Wide Web en relación a disponibilidad de servidores, energía, entre otras cuestiones (Press, 2011). Es decir, la producción de estos bienes, salvo en un inmenso número de excepciones, tiene costos sustancialmente menores a la clásica producción de bienes materiales. Aun así, el continuo desarrollo y dinámica competitiva de la WWW requiere cada vez mayores niveles de erogaciones. Y, además, en términos absolutos, el costo de producción en un proyecto colaborativo no debería tener el mismo impacto que en una empresa capitalista, dado que esta última puede enfrentar tales gastos con la valorización de su capital, es más, estos son requeridos para tal valorización. Finalmente, no deben desmerecerse diversas alternativas de financiación ampliamente difundidas en Internet como: colaboraciones, donaciones, crowdfunding, etc.

Internet presenta una diversidad de formas de producción de bienes intangibles que van desde las formas clásicas capitalistas asociadas a la propiedad privada que, criminalizan, traban y eliminan la libre circulación y reproducción de mercancías creadas (originalmente y en muchos casos para otros medios culturales) bajo su lógica de acumulación; pasando por un reducido grupo de empresas que centralizan gran parte del tráfico de la Internet mundial distribuyendo contenidos no producidos por ellos -y sus negocios- (Google, Facebook, Twitter, etc.); siguiendo por un sinfín de pequeñas empresas con múltiples y dinámicas estrategias de valorización y, finalizando, en comunidades o grupos de desarrolladores que producen código abierto por el beneficio social pero que, lamentablemente, ante "la producción en masa de productos comunicacionales se encuentran con poco espacio para sus ideas alternativas" (Pleios, 2012, p. 232).

Sin dudas la cartografía completa de Internet no puede ser dibujada, menos aún ser aprehendida. Los actores mismos son los que construyen Internet y 
se posicionan con visiones propias durante su existencia o, por el contrario, se descomponen o desaparecen (Latour y Zadunaisky, 2008, pp. 52-56). La permanencia de las plataformas en Internet es una excepción reducida en número, pero impactante en cuanto a sus conexiones. El resto de los sitios es de existencia menor, en gran medida efímera. Nada es fijo, ni siquiera lo que mantiene su existencia. ¿Es lo mismo YouTube en el 2006 que en los últimos meses?

Como hipótesis, cabe señalar que, probablemente, con el paso de los años, las clásicas formas de valorización del capital, asociadas a lo privativo y al consumo excluyente tiendan a desaparecer definitivamente. Es probable que emerja un escenario, en el cual los bienes con características gratuitas y de libre difusión dominen una Internet en la cual la totalidad de sitios y audiencias estén monopolizadas por un reducido número de gigantes capitalistas nacidos, en un principio, bajo formas de un "saber social general" (Marx, 1973). Estos actores podrán ser capaces de generar valor de cambio mediante la venta de información detallada de las necesidades y deseos de sus audiencias a empresas productoras de bienes materiales que busquen colocar sus mercancías. De este modo, el precio dejaría definitivamente de mediar directamente las relaciones sociales en Internet. En su reemplazo emergerían los paradigmas de la universalidad, la gratuidad y las comunidades cautivadas/valorizables. La Internet concebida como una red lógica descentralizada de alcance global, con el transcurrir del tiempo, atraviesa nuevas asociaciones, nuevos actores, nuevas relaciones de poder que en conjunto constituyen su colectivo (Latour y Zadunaisky, 2008, pp. 28-30).

\section{Conclusiones}

En el presente trabajo se ha intentado brindar un avance de un marco teórico actualmente en elaboración para el análisis de las nuevas tecnologías y, en especial, de Internet. Iniciamos el trabajo con la intención de presentar la interrelación e influencia continua entre sociedad y tecnología. Luego, introdujimos algunas categorías técnicas para aprehender a Internet como una serie de tecnologías digitales caracterizadas por un conjunto de capas imbricadas de carácter intangible y tangible. Por sus características intrínsecas, las capas intangibles pueden disociarse de sus soportes tangibles. A raíz de esto, se habilita la posibilidad de poder crear contenidos culturales de difusión universal evadiendo la relación capital-trabajo y la propiedad privada. La hipótesis de este trabajo es que esto genera un conflicto con las clásicas formas de valoración del capital en relación a mercados signados directamente por precios y a la propiedad privada. Nuestra conclusión es que la diversidad de realidades en la WWW es un entrecruzamiento entre las posibilidades que brindan los códigos técnicos en Internet desde sus inicios y la lógica del capital que, en cada caso particular, asume matices de ambas formas.

El número de sitios visitados en la World Wide Web hoy en día pertenece, como ya hemos mencionado, a un reducido número de empresas, las cuales ejercen su 
poder económico y tecnológico sobre producciones de bienes alternativos que puedan amenazar su posición dominante. Este tipo de conductas se suman a otras, por ejemplo, en la adquisición de empresas y la contratación de los desarrolladores del software alternativo. Estas conductas se amparan en una ínfima regulación legal que convirtió a Internet en un ámbito plenamente asimétrico.

Sin embargo, las plataformas sociales más importantes tienen buena recepción en la sociedad y son incorporadas por todo tipo de usuarios individuales o institucionales debido a sus innumerables ventajas y servicios gratuitos. Estas empresas se han convertido en parte del sustrato de la vida diaria de la humanidad conectada, son gigantes monopólicos de alcance universal (en los territorios que el capitalismo circunscribe su universalidad).

\section{Referencias}

Adorno, T. W., y Horkheimer, M. (2007). Dialéctica de la Ilustración. Ediciones AKAL.

Amariles, F., Paz, O., Russell, N., y Johnson, N. (2006). The Impacts of Community Telecentres in Rural Colombia. The Journal of Community Informatics, 3(2).

Aouragh, M. (2012). Social Media, Mediation and the Arab Revolutions. Triple C: Communication, Capitalism \& Critique. Open Access Journal for a Global Sustainable Information Society, 10(2), 518-536.

Bijker, W. E. (1997). Ofbicycles, bakelites, and bulbs: toward a theory of sociotechnical change. Cambridge, Mass: MIT Press.

Earl, J., y Kimport, K. (2011). Digitabli Enabled Social Change. Activism in the Internet Age. Cambridge, MA: MIT Press.

Ekman, M. (2012). Understanding Accumulation: The Relevance of Marx's Theory of Primitive Accumulation in Media and Communication Studies. Triple C: Communication, Capitalism \& Critique. Open Access Journal for a Global Sustainable Information Society, 10(2), 156-170.

Feenberg, A. (2010). Between reason and experience essays in technology and modernity. Cambridge, Mass.: MIT Press. Recuperado de http://site.ebrary. com/id/10392382

Finquelievich, S., y Prince, A. (2007). El (involuntario) rol social de los cibercafés (Cibercafes' (involuntary) social role). Buenos Aires: Editorial Dunken.

Fisher, E. (2012). How Less Alienation Creates More Exploitation? Audience Labour on Social Network Sites. Triple C: Communication, Capitalism \& Critique. Open Access Journal for a Global Sustainable Information Society, 10(2), 171-183. 
Gómez, R., y Barón Porras, L. F. (2010). Acceso público a Internet y cambio social: la experiencia en El Carmen de Bolívar, entre el silenciamiento y la esperanza.

Gramsci, A. (1986). Cuadernos de la cárcel. Ediciones Era.

Hall, R., y Stahl, B. (2012). Against Commodification: The University, Cognitive Capitalism and Emergent Technologies. Triple C: Communication, Capitalism $\mathcal{E}$ Critique. Open Access Journal for a Global Sustainable Information Society, 10(2), 184-202.

Hardt, M., y Negri, A. (2002). Imperio. Editorial Paidós.

Hardt, M., y Negri, A. (2006). Multitude: War and Democracy in the Age of Empire. Penguin Books, Limited.

Jameson, F. (2011). Representing Capital: a commentary of volume one. London; New York: Verso.

Jhally, y Sut. (1989). The Political Economy of Culture. En Cultural Politics in Contemporary America, 65-81. New York: Routledge.

Latour, B. (1992). Ciencia en acción: cómo seguir a los científicos e ingenieros a través de la sociedad. Labor.

Latour, B., y Zadunaisky, G. (2008). Reensamblar lo social: una introducción a la teoría del actor-red. Manantial.

Lefebvre, T. W. (1974). La producción del espacio.

Lessig, L. (2004). Cultura Libre.

Marx, K. (1972). The Eighteenih Brumaire of Louis Bonaparte. Moscú: Progress Publisher.

Marx, K. (1973). Grundrisse: Foundations of the Critique of Political Economy. Random House.

McGuigan, J. (2012). The Coolness of Capitalism Today. Triple C: Communication, Capitalism \& Critique. Open Access Journal for a Global Sustainable Information Society, 10(2), 425-438.

Miki, K. (1967). Miki Kyoshi Zenshu (Vol. 7). Tokio, Japón: Iwanami.

Mosco, V. (2010). The political economy of communication. Los Angeles: Sage.

Nixon, B. (2012). Dialectical Method and the Critical Political Economy of Culture. Triple C: Communication, Capitalism \& Critique. Open Access Journal for a Global Sustainable Information Society, 10(2), 439-456. 
Parkinson, S., y Lauzon, A. C. (2008). The Impact of the Internet on Local Social Equity: A Study of a Telecenter in Aguablanca, Columbia. MIT Press Journals, 3(4), 21-38.

Peekhaus, W. (2012). The Enclosure and Alienation of Academic Publishing: Lessons for the Professoriate. Triple C: Communication, Capitalism \& Critique. Open Access Journal for a Global Sustainable Information Society, 10(2), 577-599.

Pleios, G. (2012). Communication and Symbolic Capitalism. Rethinking Marxist Communication Theory in the Light of the Information Society. Triple C: Communication, Capitalism \& Critique. Open Access Journal for a Global Sustainable Information Society, 10(2), 230-252.

Press, E. (septiembre 12 de 2011). Google supone el 0,01 por ciento del consumo energético mundial. europapress.es. Recuperado el 14 de agosto de 2013 de http://www.europapress.es/portaltic/empresas/noticia-google-supone001-ciento-consumo-energetico-mundial-20110912162054.html

Prey, R. (2012). The Network's Blindspot: Exclusion, Exploitation and Marx's Process-Relational Ontology. Triple C: Communication, Capitalism \& Critique. Open Access Journal for a Global Sustainable Information Society, 10(2), 253-273.

Prodnik, J. (2012). A Note on the Ongoing Processes of Commodification: From the Audience Commodity to the Social Factory. Triple C: Communication, Capitalism \& Critique. Open Access Journal for a Global Sustainable Information Society, 10(2), 274-301.

Schröter, J. (2012). The Internet and «Frictionless Capitalism». Triple C: Communication, Capitalism \& Critique. Open Access Journal for a Global Sustainable Information Society, 10(2), 302-312.

Smythe, D. (1977). Communications: Blindspot of Western Marxism. Canadian Journal of Political and Social Theory, 1(3), 1-27.

Stallman, R. M. (2004). Software libre para una sociedad libre. Proyecto Editorial Traficantes de Sueños.

Surrat, C. (2001). The Internet and Social Change. Jefferson, Carolina del Norte: McFarland \& Co.

Unwin, T. (Ed.). (2009). ICT4D: Information and Communication Technology for Development. Cambridge University Press.

Vercelli, A. (2009). Repensando los bienes intelectuales comunes. Análisis socio-técnico sobre el proceso de co-construcción entre las regulaciones de derecho de autor y derecho de copia y las tecnologías digitales para su gestión (doctorado). Quilmes, Argentina: Universidad Nacional de Quilmes.

Virno, P., Estop, J. D., y Santucho, M. (2003). Gramática de la multitud: para un análisis de las formas de vida contemporáneas. Madrid: Traficantes de Sueños. 
Wallerstein, I. (1988). El capitalismo histórico. España: Siglo XXI de España Editores.

Warschauer, M. (2003). Technology and Social Inclusion: Rethinking the Digital Divide. Cambridge, MA: MIT Press.

Weber, M. (1998). La ética protestante y el espíritu del capitalismo. Ediciones AKAL.

White, M. (Agosto 12 de 2010). Clicktivism is ruining leftist activism. The Guardian. Recuperado el 30 de agosto de 2013 de http://www.theguardian. com/commentisfree/2010/aug/12/clicktivism-ruining-leftist-activism

Wittel, A. (2012). Digital Marx: Toward a Political Economy of Distributed Media. Triple C: Communication, Capitalism \& Critique. Open Access Journal for a Global Sustainable Information Society, 10(2), 313-333.

Zukerfeld, M. (2010). De niveles, regulaciones capitalistas y cables submarinos: Una introducción a la arquitectura política de Internet. Virtualis, (1), 5-21. 